\title{
Les premières grossesses humaines obtenues après détermination du sexe in vitro
}

Un article paru dans Nature le 19 avril 1990 [1], qui a fait grand bruit, annonce la réalisation de grossesses chez des couples à risque après fertilisation in vitro et " sexage " avant réimplantation. A vrai dire, on savait cette opération faisable puisque les mêmes auteurs (Handyside et al., Londres, GB) en avaient montré il y a un an la possibilité technique $(\mathrm{m} / \mathrm{s}$ $n^{\circ} 5$, vol. 5, p. 350) et qu'une démonstration analogue avait été apportée chez la souris en $1987(\mathrm{~m} / \mathrm{s}$ $n^{\circ} 10$, vol. $\left.3, p .618\right)$. Après fécondation in vitro, une cellule est prélevée au stade 6-10 cellules, l'ADN est amplifié et on détermine le sexe grâce à une séquence spécifique du chromosome $Y$. Le prélèvement n'endommage pas la culture et les embryons se développent aussi bien que les témoins. On peut en principe proposer deux types d'application de la technique : dans les maladies génétiques, reconnaître les embryons déficients; dans les maladies liées au sexe, se borner à un diagnostic de sexe et réimplanter uniquement les embryons féminins. C'est cette méthode que les auteurs ont choisie. Cinq couples à risque pour des maladies liées à l'X (adrénoleucodystrophie, Lesch-Nyhan, $X$ fragile et myopathie de Duchenne) ont suivi ce traitement. Il faut trois jours pour arriver au stade 6-10 cellules, alors que six à huit heures suffisent pour déterminer le sexe et que la réimplantation peut être réalisée le même jour. Bien que le nombre de ses cellules soit quelque peu diminué, l'implant se développe normalement. Le nombre total d'embryons examinés, 46, contenait exactement moitié de chaque sexe, et on a pu dans tous les cas obtenir au moins deux embryons femelles pour transfert. L'implantation et un début de développement eurent lieu dans tous les cas, mais deux femmes seulement avaient une grossesse en cours à 20 semaines, toutes deux ayant des jumelles dizygotes, normales aux ultrasons. Au total 4 embryons sur 17 transférés se sont développés, et cette proportion de $24 \%$ est habituelle. La méthode est donc pratiquable... mais l'expérimentation et sa publication dans Nature posent problème. Voici en effet une expérimentation humaine - réimplantation et suivi du développement d'embryons auxquels ont été prélevées des cellules in vitro - menée en l'absence de réelle étude préalable d'inocuité, ce qui est assez rare pour être signalé. Certes, de nombreux arguments tirés de l'embryologie des petits mammifères suggèrent qu'une telle manipulation de l'embryon pré-implantation ne perturbe pas le développement ultérieur... mais nous ne sommes pas des souris et on ne peut exclure $a$ priori que le développement des primates et de l'homme soit plus sensible à ce type d'intervention que celui des rongeurs. A tout le moins, une série suffisante sur les singes de laboratoire eût été, semble-t-il, un prérequis non exorbitant avant que d'autoriser des essais sans réelle justification autre que "scientifique" puisque le diagnostic prénatal tel qu'il est pratiqué aujourd'hui aurait permis, de toute façon, d'éviter la naissance d'enfants atteints dans les familles présentées par l'équipe anglaise. On peut aussi être étonné par la hâte avec laquelle ces résultats, sensationnels quant à leurs indications éthiques mais non sur le plan technique et scientifique, ont été publiées... deux grossesses gemellaires se poursuivant... pour l'instant. N'eût-il pas été sage, pour les auteurs et les éditeurs de Nature, d'attendre la naissance des enfants et leur examen biologique et clinique?
Par ailleurs, le principe même du tri d'embryons sur la base du sexe comporte un risque majeur, esquivé apparemment par les auteurs [1] comme par les commentateurs dans le même numéro [2] : que la détermination du sexe ne déborde plus tard sur un diagnostic de convenance pour choisir le sexe de l'enfant. Pour l'avenir, le même groupe tente de mettre au point des techniques de diagnostic unicellulaire pour des maladies génétiques graves [3]. Le tri d'embryons conçus par la fécondation in vitro (FIV) afin de ne réimplanter que ceux qui ne risquent pas d'être affectés pourrait, théoriquement, constituer une alternative à l'interruption de grossesse après diagnostic prénatal précoce positif. Il faudrait cependant, pour qu'une telle approche soit généralisable, que les pourcentages de succès des FIV s'améliorent significativement, alors qu'ils ne sont aujourd'hui que de l'ordre de 20 à $25 \%$.

J.C.D. A.K.

1. Handyside AH, Kontogianni EH, Hardy K, Winston RML. Pregnancies from biopsied human preimplantation embryos sexed by $\mathrm{Y}$ specific DNA amplification. Nature $1990 ; 344$ : 768-70.

2. Opinion. Embryo research. Nature 1990 ; $344: 690$.

3. Coutelle C, Williams C, Handyside AH, el al. Genetic analysis of DNA from single human ovocytes : a model for preimplantation diagnosis of cystic fibrosis. Br Med J 1989 ; 299 : 22-4. 